\title{
Identifying familial hypercholesterolemia in an early onset ischemic cerebrovascular disease patient and the cascade screening in the pedigree: a case report
}

\author{
Yuan Yuan ${ }^{1 \# \wedge}$, Xiaoyan Zhao ${ }^{2 *}$, Xiangyun Teng ${ }^{1}$, Yunfeng Zhang $^{2}$ \\ ${ }^{1}$ Department of Neurophysiology and Neuropharmacology, Institute of Special Environmental Medicine and Co-innovation Center of \\ Neuroregeneration, Nantong University, Nantong, China; ${ }^{2}$ Department of Neurology, Affiliated Hospital of Nantong University, Nantong, China \\ \#These authors contributed equally to this work. \\ Correspondence to: Yunfeng Zhang. Department of Neurology, Affiliated Hospital of Nantong University, Nantong 226001, China. \\ Email: dannychangnt@sina.com.
}

\begin{abstract}
Familial hypercholesterolemia $(\mathrm{FH})$ is one of the most common inherited metabolic disorders characterized by elevated low-density lipid cholesterol (LDL-C) levels that lead to coronary artery disease at an early age and a low occurrence of cerebrovascular disease. Low-density lipoprotein receptor $(L D L R)$ gene mutation is the most common cause of $\mathrm{FH}$. Here, we report a case of a 47-year-old woman who had multiple carotid artery stenosis and brain ischemic foci, an elevated level of LDL-C, underwent eyelid xanthoma excision, and a family history of hyperlipidemia. Thereafter, she was diagnosed with $\mathrm{FH}$ according to the Dutch Lipid Clinical Network criteria and whole genome sequencing revealed compound heterozygous LDLR mutations. However, she denied a history of coronary heart disease (CAD). The patient underwent stenting of the right subclavicular artery and right internal carotid artery in our hospital. Lipid-lowering drugs were also administered to prevent stroke recurrence. During a 3-year follow-up, the blood lipid level of the patient reduced, and the condition of intracranial and extracranial vascular stenosis improved. Furthermore, a cascade screening was performed in her pedigree, and 7/9 family members were found to have elevated LDL-C, 6/7 were found to carry one of the two LDLR variants detected in the proband, and in $4 / 6$, the carotid intima-media thickness was $\geq 1 \mathrm{~mm}$, which was predicted as a high risk factor of cerebrovascular disease. Her relatives with high risks of cardiovascular or cerebrovascular diseases have been under lipid monitoring and management of risk factors since then. To date, no cardiovascular or cerebrovascular event has been reported. In conclusion, this case reminds us to consider FH screening in early-onset stroke or transient ischemic attack patients with elevated LDL-C level. Our report also demonstrates the beneficial role of genetic testing and cascade screening in the relatives of $\mathrm{FH}$ patients.
\end{abstract}

Keywords: Familial hypercholesterolemia (FH); early onset cerebrovascular disease; cascade screening; whole genome sequencing; case report

Submitted Aug 03, 2020. Accepted for publication Nov 15, 2020.

doi: $10.21037 /$ atm-20-5600

View this article at: http://dx.doi.org/10.21037/atm-20-5600

\footnotetext{
^ ORCID: 0000-0001-5880-4384.
} 


\section{Introduction}

Familial hypercholesterolemia $(\mathrm{FH})$ is a common genetic disorder characterized by elevated cholesterol concentrations, especially low-density lipid cholesterol (LDL-C), in early life, and is associated with premature cardiovascular disease. Mutations in four genes have been reported to be responsible for an estimated $70-95 \%$ of FH: LDL receptor $(L D L R)$, apolipoprotein B $(A p o B)$, proprotein convertase subtilin/kexin 9 (PCSK9), and lowdensity lipoprotein receptor adaptor protein (LDLRAP). Additionally, $L D L R$ mutation has been shown to be the most common cause. Its inheritance is in an autosomal codominant way in most cases. $\mathrm{FH}$ increases the risk of premature coronary heart disease (CAD), which may manifest as angina and myocardial infarction; stroke occurs more rarely (1-4).

Here, we report the case of a 47-year-old woman who had multiple carotid artery stenosis, ischemic brain foci, and elevated plasma LDL-C level, but no sign of CAD, who was diagnosed with $\mathrm{FH}$ according to Dutch Lipid Clinical Network (DLCN) criteria and carried compound heterozygous pathogenic $L D L R$ variants. Based on her diagnosis, a cascade screening was performed in her pedigree. We present the following case in accordance with the CARE reporting checklist (available at http://dx.doi. org/10.21037/atm-20-5600).

\section{Case presentation}

All procedures performed in studies involving human participants were in accordance with the Helsinki Declaration (as revised in 2013), and this study was approved by the ethics committee of the Affiliated Hospital of Nantong University (approval number: 2019-K092). Written informed consent was obtained from the patient for publication of this study and any accompanying images.

The patient was a 47-year-old woman who visited our hospital in January 2017 with a complaint of numbness and weakness in the left limb for more than one month. When we inquired about her medical history, the patient mentioned that she had a history of "hyperlipidemia" nine months prior and had not received regular treatment. She had a history of hypertension for more than 10 years and took valsartan orally to control her blood pressure. The patient also had a history of eyelid xanthoma excision 20 years ago. When inquired about her family history, she mentioned that her mother died from a sudden cerebral hemorrhage 28 years ago; her brother, half-sister, and their offspring, all had hyperlipidemia. However, she denied a history of CAD. After admission, the patient's physical examination showed a slightly sluggish response, mild hemiplegia in the left limb, and a slight decreased response to pain and touch. Her total cholesterol, triglyceride, LDL-C, and head and neck angiography were then examined (Table 1 and Figure 1). A

Table 1 Clinical and genetic characteristics of the family members in the cascade screening

\begin{tabular}{|c|c|c|c|c|c|c|c|c|c|c|c|}
\hline No. & Sex & Age & BMI & $\mathrm{TC}(\mathrm{mM})$ & $\mathrm{TG}(\mathrm{mM})$ & HDL-C (mM) & LDL-C (mM) & ICD & c-IMT & DLCN & LDLR variant \\
\hline III $4^{\ddagger}$ & $\mathrm{F}$ & 49 & 25.3 & 8.3 & 1.5 & 1.3 & $8.5^{\dagger}$ & Y & $Y$ & de & $\mathrm{v} 1+\mathrm{v} 2$ \\
\hline II1 & $M$ & 52 & 21.2 & 9.0 & 0.8 & 1.4 & 6.5 & $\mathrm{~N}$ & Y & $\mathrm{pr}$ & $\mathrm{v} 1$ \\
\hline II5 & $\mathrm{F}$ & 68 & 25.0 & 7.2 & 1.5 & 1.4 & 4.8 & $\mathrm{~N}$ & $\mathrm{~N}$ & po & $\mathrm{v} 1$ \\
\hline III2 & M & 28 & 24.3 & 5.9 & 1.2 & 1.2 & 4.1 & $\mathrm{~N}$ & $\mathrm{~N}$ & po & $\mathrm{N}$ \\
\hline III3 & $\mathrm{F}$ & 26 & 17.1 & 6.7 & 1.2 & 1.0 & 4.7 & $\mathrm{~N}$ & $\mathrm{~N}$ & po & $\mathrm{v} 1$ \\
\hline IIII5 & $M$ & 17 & 16.0 & 5.7 & 0.5 & 1.4 & 3.9 & $\mathrm{~N}$ & $\mathrm{~N}$ & po & v2 \\
\hline IIII6 & $M$ & 43 & 29.3 & 8.9 & 2.3 & 0.8 & 6.9 & $\mathrm{~N}$ & Y & $\mathrm{pr}$ & $\mathrm{v} 1$ \\
\hline IIII9 & $\mathrm{F}$ & 40 & 22.9 & 9.0 & 1.5 & 1.2 & 6.8 & $\mathrm{~N}$ & Y & $\mathrm{pr}$ & $\mathrm{v} 1$ \\
\hline IV3 & M & 19 & 31.9 & 8.2 & 1.1 & 1.0 & 6.2 & $\mathrm{~N}$ & Y & po & $\mathrm{v} 1$ \\
\hline IV4 & M & 17 & 26.2 & 3.6 & 0.9 & 1.0 & 2.2 & $\mathrm{~N}$ & $\mathrm{~N}$ & un & $\mathrm{N}$ \\
\hline
\end{tabular}

${ }^{\dagger}$, represents the estimated value before taking medicine calculated by measured value divided 0.7 ; ${ }^{\ddagger}$, proband; No., Pedigree chart coding number; BMI, body mass index; TC, total cholesterol; TG, triglycerides; HDL-C, high density lipoprotein cholesterol; LDL-C, low density lipoprotein cholesterol; ICD, ischemic cerebrovascular disease, when the patient had early-onset ICD, determined by male $\leq 55$ years old, female $\leq 60$ years old, it was yes $(\mathrm{Y})$, otherwise it was no $(\mathrm{N})$; c-IMT, carotid intima-media thickness, when the value $\geq 1 \mathrm{~mm}$, it was yes $(\mathrm{Y})$, otherwise it was no (N); DLCN, Dutch Lipid Clinical Network criteria; de, definite; pr, probable; po, possible; un, unlikely; LDLR variant, sanger sequencing result of the two LDLR variants, v1 and v2 represent variant 1 and variant 2 respectively; N, no variant; F, female; M, male. 

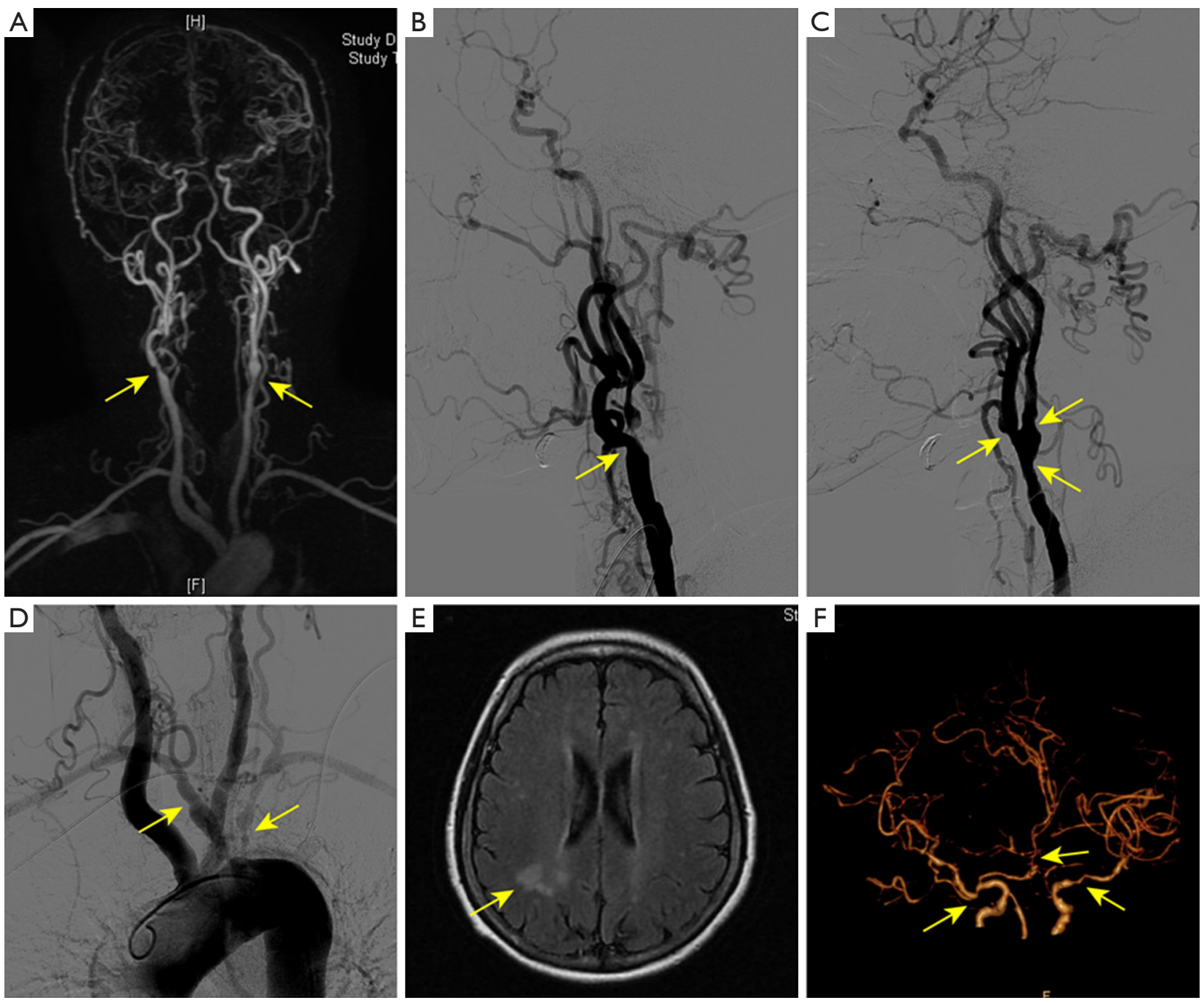

Figure 1 Imaging of the carotid artery stenosis and brain ischemic foci of the proband. (A) Multiple stenosis of bilateral distal common carotid artery detected by magnetic resonance angiography. Severe stenosis of the right distal common carotid artery (B) and the subtotal occlusion in the left distal common carotid artery, proximal internal carotid artery, external carotid artery (C), and multiple stenosis of bilateral proximal subclavicular artery (D) detected by digital subtraction angiography. Multiple ischemic foci are found in bilateral basal ganglia, and white matter hemioval center as detected by cranial magnetic resonance imaging; abnormal signals are seen in the right occipital lobe (E). (F) The intracranial segment of computed tomography angiography in the head and neck shows multiple intracranial vascular stenosis. The carotid artery stenosis and brain ischemic foci are indicated by yellow arrows.

review of cranial computed tomography angiography in our hospital suggested that there were mixed plaques and mild to moderate stenosis in the wall of bilateral common carotid artery, and segmental stenosis in bilateral middle cerebral artery and anterior cerebral artery. Thereafter, stenting of the right subclavicular artery and the right internal carotid artery was performed in our hospital, and longterm oral antiplatelet aggregation and lipid-lowering drugs (atorvastatin $40 \mathrm{mg} /$ day and ezetimibe $10 \mathrm{mg} /$ day) were used to prevent recurrence of stroke. During a 3-year followup, the blood lipid level of the patient reduced, and the condition of intracranial and extracranial vascular stenosis improved. Moreover, no evidence of arterial calcification was observed during the follow-up.

This patient was not diagnosed with obesity, diabetes, or other conditions that could lead to secondary 


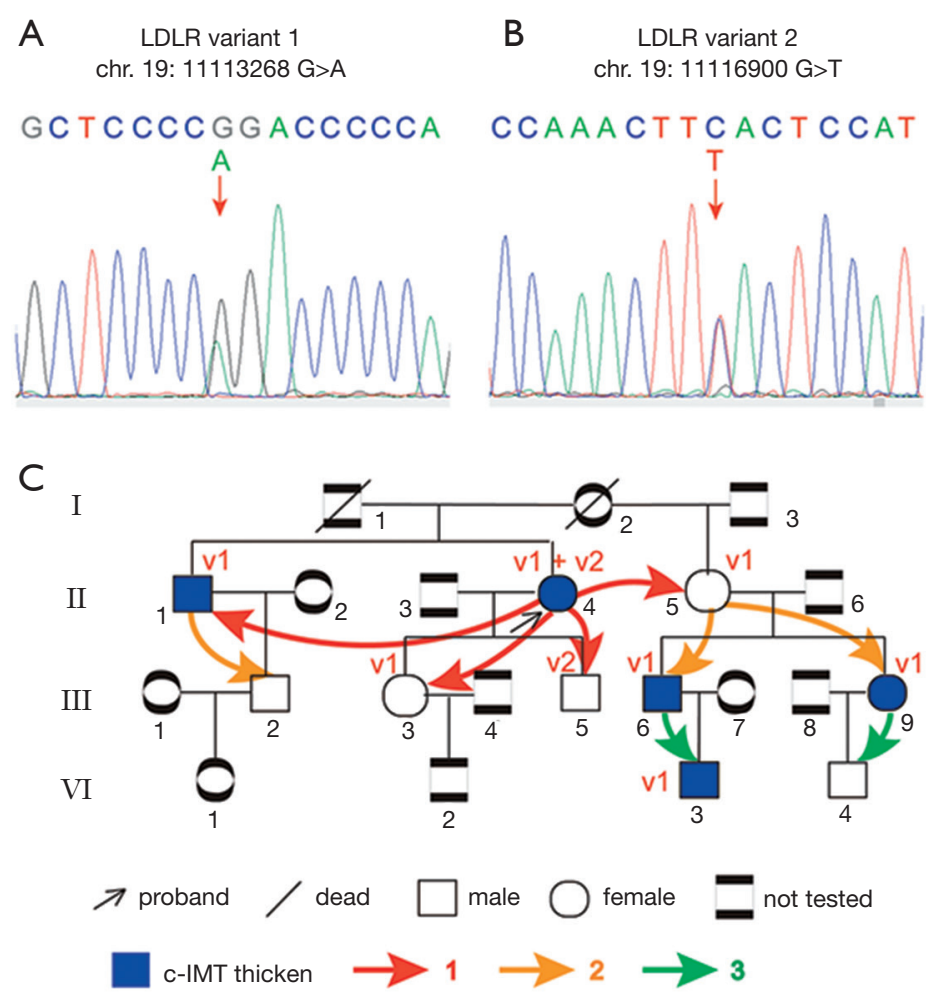

Figure 2 Validation of $L D L R$ variants in the proband through Sanger sequencing, and the gene test results in the pedigree through cascade screening. (A,B) show the validation results of Sanger sequencing of the $L D L R$ variants 1 and 2 in the proband discovered through whole genome sequencing, respectively. (C) The cascade screening in the pedigree (shown by the colorful arrows), the c-IMT (filled with blue), and $L D L R$ variant test results marked with v1 and v2; v1 represents $L D L R$ variant 1, while v2 represents $L D L R$ variant 2, which were found in the proband.

hypercholesterolemia or phytosterolemia, nor intook extra cholesterol. Her clinical features and family history indicated the possibility of FH. FH was diagnosed according to the DLCN criteria (5), her score was 11, and was classified as definite $\mathrm{FH}$. To further confirm genetic analysis and to identify disease-causing mutations, whole genome sequencing was performed. $90.41 \mathrm{G}$ raw data and $89.26 \mathrm{G}$ clean data were obtained on the Illumina sequencing platform. A total of 3,662,148 single nucleotide variations, 891,303 insertions and deletions, 502 copy number variations, and 1,500 structural variations were detected. Among these variations, two mutations in $L D L R$ drew our attention, since $L D L R$ mutations are responsible for about $70 \%$ of $\mathrm{FH}$ occurrence (1). One of the mutations was chr. 19: $11113268 \mathrm{G}>\mathrm{A}$ (defined as variant 1); it is represented as NM_000527.5 (LDLR): c.1187-10G>A in the ClinVar database (Variant ID: 226349). This variant changes a single nucleotide in intron 8 of LDLR mRNA and is predicted to alter mRNA splicing. The other mutation was chr. 19: $11116900 \mathrm{C}>\mathrm{T}$ (defined as variant 2), represented as NM_000527.5 (LDLR): c.1747C>T (p. His583Tyr, or p.H583Y) in ClinVar (Variant ID: 200921). Both mutations are interpreted as pathogenic/likely pathogenic according to previous research. The two variants were confirmed by Sanger sequencing (Figure $2 A, B$ ). Based on the gene sequencing results, the patient's condition was unequivocally $\mathrm{FH}$.

Subsequently, cascade screening was performed among the proband's family members. Three rounds of cascade screening were conducted (Figure 2C), and the evaluation of $\mathrm{FH}$ was performed according to the DLCN criteria. The first round tested the first-degree relatives of the proband (II4), including her brother (II1), half-sister (II5), her daughter (III3), and son (III5). II1 was classified as a 
probable FH patient, while II5, III3, and III5 were classified as possible FH patients. In the second round, we assessed the first-degree relatives of II1 and II5. We tested the son of II1 (III2) and the son (III6) and daughter (III9) of II5, and the screening results were possible, probable, and probable FH patients, respectively. In the third round, we screened the son of III6 (IV3) and III9 (IV4); the evaluation results were possible and unlikely FH patient, respectively. All the cascade screening results are summarized in Table 1.

Furthermore, the two pathogenic $L D L R$ variants carried by the proband were detected in her pedigree through high fidelity PCR and Sanger sequencing. Unfavorably, 6/9 and $1 / 9$ of the relatives were found to carry $L D L R$ variants 1 and 2, respectively (Table 1 and Figure 2C). Except for III2, who was classified as a possible $\mathrm{FH}$ patient but did not carry any of the two $L D L R$ pathogenic variants, the diagnosis results according to the DLCN criteria were consistent with the gene test results. We have suggested lipid monitoring, risk factor management, and lifestyle guidance for her relatives with high risks of cardiovascular or cerebrovascular diseases. To date, no event related to either of these diseases has been reported in her pedigree.

\section{Discussion}

The relationship between $\mathrm{FH}$ and ischemic cerebrovascular disease is controversial. In the present case, the index patient was diagnosed with FH according to the DLCN criteria and genetic test results. She presented with the clinical features of ischemic cerebrovascular disease at an early age, while denying the sign of $\mathrm{CAD}$, which is the most common manifestation of $\mathrm{FH}$. Although stroke occurs more rarely than $\mathrm{CAD}$ in patients with $\mathrm{FH}$, cases like ours may not be that uncommon. In a study on patients with acute ischemic stroke or transient ischemic attack (TIA), based on the DLCN algorithm, the frequencies of potential $\mathrm{FH}$ and probable/definite $\mathrm{FH}$ were $11.5 \%$ and $1.3 \%$, respectively. The prevalence was even higher in patients with early onset of stroke/TIA manifestation (before the age of 55 in men or 60 in women, $13.1 \%$ or $3.1 \%$, respectively). Moreover, in two-thirds of the patients with probable/definite and possible $\mathrm{FH}$, stroke or TIA was the initial clinical manifestation of the disease. Therefore, the authors suggest to consider FH screening as part of the usual diagnostic work-up in stroke or TIA patients (6). Moreover, aggressive treatment has been recommended for $\mathrm{FH}$ patients with early-onset of TIA or stroke (7). Additionally, a higher prevalence of cerebral small vessel disease in in patients with $\mathrm{FH}$ compared to that in healthy controls ( $25 \%$ versus $5.7 \%)$ was reported in a study based on $7 \mathrm{~T}$ magnetic resonance imaging. Therefore, the authors recommended that attention should be paid to not only the development of CAD but also cerebral vascular disease in patients with FH (8). Further studies are needed in the future to improve the detection rate of $\mathrm{FH}$ in patients with ischemic stroke or TIA so that they can be treated before the onset of CAD.

FH is currently substantially under-diagnosed and undertreated worldwide, despite being the most common genetic disorder with a prevalence of $1 / 200$ to $1 / 250$ (9). Only $10 \%$ of the $\mathrm{FH}$ population are diagnosed and adequately treated because of the lack of awareness of FH among the public and medical community. To improve the diagnosis and treatment of $\mathrm{FH}$, a global call to action was made by the Global Familial Hypercholesterolemia Community to provide public policy recommendations covering awareness, advocacy, screening, testing and diagnosis, treatment, family-based care, registries, research, and cost and value (9). Among these aspects, diagnosis is one of the cornerstones. Until now, several clinical criteria including Simon Broome (from the UK), DLCN (from the Netherlands), Make Early Diagnosis to Prevent Early Death (MEDPED) (from the USA), and the recent Montreal-FH-SCORE have been applied in FH diagnosis. All of these criteria comprise of several prognostic scores including patient's lipid levels, cutaneous stigmata, and personal and family history of CAD. Moreover, owing to the rapid improvement and wide availability of molecular diagnosis methods, identification of a pathogenic variant in a gene known to be associated with FH has become the gold standard for FH diagnosis (10).

Although genetic testing provides great help in the diagnosis of $\mathrm{FH}$, a discrepancy in clinical and genetic $\mathrm{FH}$ diagnosis exists. In the present case, one relative of the proband who was diagnosed with possible $\mathrm{FH}$ based on DLCN criteria did not carry any of the $L D L R$ pathogenic mutations found in the proband. This situation is not rare. It has been reported that in $28-80 \%$ of $\mathrm{FH}$ patients examined over clinical criteria alone, depending on the diagnostic algorithm and the method of analysis, genetic testing provided confirmation (11). Some patients with a clinical diagnosis of definite $\mathrm{FH}$ have no mutations in the genes associated with the disease but still have a high cardiovascular risk $(12,13)$. A polygenic etiology is the most probable reason for majority of individuals to be clinically diagnosed with $\mathrm{FH}$ while no known pathogenic variant could be identified (14). Based on this situation, $\mathrm{FH}$ has been proposed as a syndrome that is composed of patients with or without detected disease- 
causing mutations. Early and intensive lipid-lowering therapy is recommended for all patients with FH (12). Notably, a confirmed molecular diagnosis reportedly increases lipidlowering therapy adherence (11).

Cascade screening of $\mathrm{FH}$ is much more cost-effective than universal screening. In two large population-based studies consisting of 76,751 individuals, an FH-causing variant was identified only in $1.7-2.5 \%$ of subjects with LDL-C levels over $4.9 \mathrm{mmol} / \mathrm{L}(190 \mathrm{mg} / \mathrm{dL})$; therefore, genetic testing of $\mathrm{FH}$-causing mutations has a poor yield in unselected patients with elevated levels of LDL-C as the sole criterion (11). Meanwhile, $44.76 \%$ (30-60\%) new cases on average when using a cascade testing strategy, and 4.11 versus 1.06 new cases per index case were found when active sample collection was used compared to the collection at the clinic, showing that active cascade screening is a cost-effective way to screen patients with $\mathrm{FH}$ (15). In the present case, we performed active cascade screening in the proband's family and found that 7/9 of her family members had elevated LDL-C levels, and 6/7 carried one of the $L D L R$ variants found in the proband. Moreover, 4 family members with $c$-IMT $\geq 1 \mathrm{~mm}$ carried LDLR variant 1 . Therefore, our case demonstrated the beneficial role of active cascade screening. Furthermore, early unequivocal diagnosis helps family members in the prevention of cerebrovascular disease and/or CAD at an early age. Since III3 has been validated to carry $L D L R$ variant 1 , a genetic test is expected to be performed for her 5-year-old son (IV2) soon, and she was grateful to us for the active screening and early diagnosis of her son.

Appropriate treatment is essential to prevent or delay the onset of cardiovascular disease in $\mathrm{FH}$ patients. The optimal LDL-C goal for lipid-lowering therapy recommended by international guidelines is $<100 \mathrm{mg} / \mathrm{dL}(<2.6 \mathrm{mmol} / \mathrm{L})$ for adults or $<70 \mathrm{mg} / \mathrm{dL}(<1.5 \mathrm{mmol} / \mathrm{L})$ for adults with coronary heart disease or diabetes, and $<135 \mathrm{mg} / \mathrm{dL}$ $(<3.5 \mathrm{mmol} / \mathrm{L})$ for children, or at least a $50 \%$ reduction in LDL-C levels $(16,17)$. Traditional treatments for FH are statins (3-hydroxy-3-methylglutaryl-CoA reductase inhibitors), ezetimibe (a selective transport inhibitor of cholesterol and phytosterol in the small intestinal mucosa), and apheresis. Lomitapide (an inhibitor of microsomal triglyceride transfer protein), mipomersen (an antisense molecule that targets the mRNA encoding APOB-100 produced by the liver), bile acid sequestrant, and niacin have also been used for LDL-C reduction in homozygous FH patients $(7,17,18)$. The approval of PCSK9 inhibitors in 2015 started a new era for cardiovascular disease prevention in patients with FH. Evolocumab, a PCSK9 inhibitor, is proven to be well tolerated and effectively reduces plasma LDL-C levels in homozygous FH and severe heterozygous FH patients over a median of 4.1 years (19). Besides, several experimental drugs are under clinical trials. Evinacumab, being a very promising one, is a monoclonal antibody that binds to ANGPTL3 with the aim to further reduce the level of LDL-C in homozygous FH patients (20). With advancement in research, more treatment options are expected to be available for patients with $\mathrm{FH}$ in the future.

In conclusion, this case reminds us of the possibility of $\mathrm{FH}$ in patients with early onset of cerebrovascular disease. Further, when a patient is diagnosed with $\mathrm{FH}$, a cascade screening including clinical and genetic tests should be strongly recommended based on pedigree.

\section{Acknowledgments}

We appreciate the language editing service provided by Elsevier Webshop.

Funding: None.

\section{Footnote}

Reporting Checklist: The authors have completed the CARE reporting checklist. Available at http://dx.doi.org/10.21037/ atm-20-5600

Conflicts of Interest: All authors have completed the ICMJE uniform disclosure form (available at http://dx.doi. org/10.21037/atm-20-5600). The authors have no conflicts of interest to declare.

Ethical Statement: The authors are accountable for all aspects of the work in ensuring that questions related to the accuracy or integrity of any part of the work are appropriately investigated and resolved. All procedures performed in studies involving human participants were in accordance with the Helsinki Declaration (as revised in 2013), and this study was approved by the ethics committee of the Affiliated Hospital of Nantong University (approval number: 2019-K092). Written informed consent was obtained from the patient for publication of this study and any accompanying images.

Open Access Statement: This is an Open Access article distributed in accordance with the Creative Commons Attribution-NonCommercial-NoDerivs 4.0 International 
License (CC BY-NC-ND 4.0), which permits the noncommercial replication and distribution of the article with the strict proviso that no changes or edits are made and the original work is properly cited (including links to both the formal publication through the relevant DOI and the license). See: https://creativecommons.org/licenses/by-nc-nd/4.0/.

\section{References}

1. Singh S, Bittner V. Familial hypercholesterolemia-epidemiology, diagnosis, and screening. Curr Atheroscler Rep 2015;17:482.

2. Defesche JC, Gidding SS, Harada-Shiba M, et al. Familial hypercholesterolaemia. Nat Rev Dis Primers 2017;3:17093.

3. Benito-Vicente A, Uribe KB, Jebari S, et al. Familial Hypercholesterolemia: The Most Frequent Cholesterol Metabolism Disorder Caused Disease. Int J Mol Sci 2018;19:3426.

4. Akioyamen LE, Tu JV, Genest J, et al. Risk of Ischemic Stroke and Peripheral Arterial Disease in Heterozygous Familial Hypercholesterolemia: A Meta-Analysis. Angiology 2019;70:726-36.

5. Chan DC, Pang J, Hooper AJ, et al. A Comparative Analysis of Phenotypic Predictors of Mutations in Familial Hypercholesterolemia. J Clin Endocrinol Metab 2018;103:1704-14.

6. Toell T, Mayer L, Pechlaner R, et al. Familial hypercholesterolaemia in patients with ischaemic stroke or transient ischaemic attack. Eur J Neurol 2018;25:260-7.

7. Milionis H. Stroke patients with familial hypercholesterolemia: need for detection and aggressive treatment. Eur J Neurol 2018;25:211-2.

8. Todate $\mathrm{Y}$, Uwano I, Yashiro $\mathrm{S}$, et al. High Prevalence of Cerebral Small Vessel Disease on 7T Magnetic Resonance Imaging in Familial Hypercholesterolemia. J Atheroscler Thromb 2019;26:1045-53.

9. Representatives of the Global Familial Hypercholesterolemia C, Wilemon KA, Patel J, et al. Reducing the Clinical and Public Health Burden of Familial Hypercholesterolemia: A Global Call to Action.

Cite this article as: Yuan $\mathrm{Y}$, Zhao $\mathrm{X}$, Teng $\mathrm{X}$, Zhang $\mathrm{Y}$. Identifying familial hypercholesterolemia in an early onset ischemic cerebrovascular disease patient and the cascade screening in the pedigree: a case report. Ann Transl Med 2021;9(2):180. doi: 10.21037/atm-20-5600
JAMA Cardiol 2020;5:217-29.

10. Moldovan V, Banescu C, Dobreanu M. Molecular diagnosis methods in familial hypercholesterolemia. Anatol J Cardiol 2020;23:120-7.

11. Lee S, Akioyamen LE, Aljenedil S, et al. Genetic testing for familial hypercholesterolemia: Impact on diagnosis, treatment and cardiovascular risk. Eur J Prev Cardiol 2019;26:1262-70.

12. Masana L, Ibarretxe D, Rodriguez-Borjabad C, et al. Toward a new clinical classification of patients with familial hypercholesterolemia: One perspective from Spain. Atherosclerosis 2019;287:89-92.

13. Trinder M, Francis GA, Brunham LR. Association of Monogenic vs Polygenic Hypercholesterolemia With Risk of Atherosclerotic Cardiovascular Disease. JAMA Cardiol 2020;5:390-9.

14. Talmud PJ, Shah S, Whittall R, et al. Use of lowdensity lipoprotein cholesterol gene score to distinguish patients with polygenic and monogenic familial hypercholesterolaemia: a case-control study. Lancet 2013;381:1293-301.

15. Lee C, Rivera-Valerio M, Bangash H, et al. New Case Detection by Cascade Testing in Familial Hypercholesterolemia: A Systematic Review of the Literature. Circ Genom Precis Med 2019;12:e002723.

16. Perez de Isla L, Alonso R, Watts GF, et al. Attainment of LDL-Cholesterol Treatment Goals in Patients With Familial Hypercholesterolemia: 5-Year SAFEHEART Registry Follow-Up. J Am Coll Cardiol 2016;67:1278-85.

17. Raal FJ, Hovingh GK, Catapano AL. Familial hypercholesterolemia treatments: Guidelines and new therapies. Atherosclerosis 2018;277:483-92.

18. Duell PB, Fazio S. Aggressive Treatment for Severe Forms of Familial Hypercholesterolemia. J Am Coll Cardiol 2020;75:575-7.

19. Santos RD, Stein EA, Hovingh GK, et al. LongTerm Evolocumab in Patients With Familial Hypercholesterolemia. J Am Coll Cardiol 2020;75:565-74.

20. Raal FJ, Rosenson RS, Reeskamp LF, et al. Evinacumab for Homozygous Familial Hypercholesterolemia. N Engl J Med 2020;383:711-20. 